\title{
Parity-Violating Electron Scattering: New Results and Future Prospects
}

\author{
Krishna S. Kumar \\ Department of Physics, University of Massachusetts, Amherst, MA 01003
}

\begin{abstract}
We discuss the status and prospects of an experimental program of parity-violating asymmetry measurements in the scattering of longitudinally polarized electrons off unpolarized fixed targets. One thrust is the measurements of nucleon neutral weak form factors at intermediate four-momentum transfer $\left(0.1<Q^{2}<1\right)(\mathrm{GeV} / \mathrm{c})^{2}$ which provide information about the role of virtual strange quarks on the charge and current distributions inside nucleons. A new topic is the elastic neutral weak amplitude from scattering off a heavy spinless nucleus, which is sensitive to the presence of a neutron skin. Finally, we discuss the neutral current elastic amplitude at very low $Q^{2}$, which allows precision measurements of the weak mixing angle at low energy and is thus sensitive to new physics at the $\mathrm{TeV}$ scale. The physics implications of recent results, potential measurements from experiments under construction as well as new ideas at future facilities are discussed.
\end{abstract}

Keywords: Parity Violation, Weak Neutral Current Interactions

PACS: $12.15 . \mathrm{Mm}$ Neutral Currents 11.30.Er C, P, T and other discrete symmetries

\section{INTRODUCTION}

Nearly 50 years ago, soon after the discovery of parity violation in beta decay, Zel'dovich speculated that there might be an analogous parity violating neutral current interaction[1]. He noted that if such an interaction existed, then parity violation would be manifested in lepton-nucleon scattering due to the interference between the weak and electromagnetic amplitudes. He predicted that if one scatters longitudinally polarized electrons off unpolarized protons and flipped the sign of the beam polarization, the fractional difference in the cross-section would be:

$$
A_{\mathrm{PV}} \equiv \frac{\sigma_{R}-\sigma_{L}}{\sigma_{R}+\sigma_{L}} \simeq \frac{\left|A_{Z}\right|}{\left|A_{\gamma}\right|} \simeq \frac{G_{F} Q^{2}}{4 \pi \alpha} \simeq 10^{-4} Q^{2}
$$

For typical fixed target experiments, $A_{P V}$ ranges from roughly $10^{-4}$ to as small as $10^{-7}$. In the mid-seventies, parity violation in deep inelastic electron nucleon scattering was first observed at SLAC[2], from which the electron-quark weak neutral current coupling could be extracted. The measurement was an important test of the $\mathrm{SU}(2) \times \mathrm{U}(1)$ gauge theory of electroweak interactions, and the extracted value of the electroweak mixing angle $\sin ^{2} \theta_{W}$ matched the corresponding value obtained from neutral current neutrino scattering experiments.

Over the past 20 years, the experimental techniques employed to measure these tiny left-right asymmetries have been steadily refined such that statistical errors approaching 0.01 parts per million (ppm) and systematic errors of a few parts per billion (ppb) are possible[3]. Depending on the choice of target and kinematic variables, this has

\footnotetext{
CP870, Intersections of Particle and Nuclear Physics: $9^{\text {th }}$ Conference, edited by T. M. Liss

(c) 2006 American Institute of Physics 978-0-7354-0368-0/06/\$23.00
} 
facilitated measurements in several important physics topics, such as many-body nuclear physics, nucleon structure and searches for physis beyond the standard model at the $\mathrm{TeV}$ scale. In this review, we discuss the principal physics thrusts of the current experimental program, describe the status of ongoing experiments and speculate on future directions of this research.

\section{STRANGENESS IN NUCLEONS}

Over the past decade, several experimental programs have focused on probing for the manifestations of strangeness in nucleon properties, such as mass, spin, momentum, magnetic moment and charge radius. A clean measurement of the contribution of strange quarks to any of these properties would be a dramatic proof of non-trivial dynamics of sea quarks inside nucleons, providing a new window into nonperturbative QCD.

There are some indications that the strange quarks might contribute to the mass (via $\pi$-proton scattering measurements) and the spin (via spin-dependent deep inelastic scattering measurements). While these experiments are sensitive to the strange scalar and axial vector matrix elements, parity violating elastic electron scattering can access vector strangeness matrix elements sensitive to the the contribution of strange quarks to nucleon charge and magnetic moment distributions $[4,5]$.

Elastic electron nucleon electromagnetic scattering is well described by the Dirac and Pauli (or equivalently the Sachs electric and magnetic) form factors. One can introduce equivalent neutral weak form factors that would be accessible in parity violating elastic electron scattering. If one assumes the validity of the standard model (weak isospin symmetry) and charge symmetry and that only three flavors are active, then one needs three elastic electroweak electron nucleon amplitudes to achieve flavor separation[6]. Thus, for a given value of $Q^{2}$, if the proton and neutron electromagnetic form factors are well measured, the measurement of the neutral weak form factors at the same value of $Q^{2}$ allows the extraction of the strange form factors.

The exact calculation of the strange form factors from QCD is currently difficult since it involves nonperturbative dynamics of sea quarks. Various model approaches are used, such as chiral perturbation theory, quark models, lattice gauge theory, Skyrme models and dispersion relations [7, 8]. In order to comprehensively probe for non-zero strange quark effects in the vector form factors, measurements over the range $0.1<Q^{2}<1$ $(\mathrm{GeV} / \mathrm{c})^{2}$, as well as forward and backward angle measurements off ${ }^{1} \mathrm{H},{ }^{2} \mathrm{H}$ and ${ }^{4} \mathrm{He}$ targets, are required.

\section{Current status and new results}

Four experimental program have reported $A_{\mathrm{PV}}$ measurements[9, 11, 12, 10, 13]. All data are mutually consistent. While no single measurement has yet to establish a statistically significant signal for strange form factors, there are two regions where there are hints of non-zero values, one at $Q^{2} \sim 0.1 \mathrm{GeV}^{2}$ and one at $Q^{2} \sim 0.6 \mathrm{GeV}^{2}$, albeit with marginal statistical significance. This is the focus of current and future measurements. 
Most recently, the HAPPEX experiment has reported new measurements of the neutral weak form factors with ${ }^{1} \mathrm{H}$ and ${ }^{4} \mathrm{He}$ targets at $Q^{2} \sim 0.1 \mathrm{GeV}^{2}$. With a beam energy $E_{b}$ of $3 \mathrm{GeV}$ and a mean scattering angle of $6^{\circ}$, preliminary results were reported at the Dallas meeting of the American Physics Society in April 2006. The ${ }^{4} \mathrm{He}$ result $\left(E_{b}=2.75\right.$ $\mathrm{GeV})$ is $A_{\mathrm{PV}}=(+6.43 \pm 0.23$ (stat) \pm 0.22 (syst) $)$ ppm where the standard model expectation with zero strange form factors was $6.37 \mathrm{ppm}$. The ${ }^{1} \mathrm{H}$ result $\left(E_{b}=3.18 \mathrm{GeV}\right)$ is $A_{\mathrm{PV}}=(-1.58 \pm 0.12$ (stat) \pm 0.04 (syst) $)$ ppm, where the expectation was $-1.64 \pm 0.04$ ppm. Combined with previous HAPPEX data[13], this allows the extraction at a common $Q^{2}=0.1 \mathrm{GeV}^{2}$ of $G_{M}^{s}=0.12 \pm 0.24$ and $G_{E}^{s}=-0.002 \pm 0.017$. One can also combine all experimental data below $Q^{2}=0.2 \mathrm{GeV}^{2}$, which are mutually consistent within quoted uncertainties, to obtain the values $G_{M}^{s}=0.28 \pm 0.20$ and $G_{E}^{s}=-0.006 \pm 0.016$.

\section{Outlook}

While earlier measurements had suggested non-zero strange form factors, the new HAPPEX results leave little room for strangeness dynamics at low $Q^{2}$, as suggested by a couple of recent theoretical calculations $[14,15]$. The A4 and G0 experiments are in the process of collecting data at backward angles on both ${ }^{1} \mathrm{H}$ and ${ }^{2} \mathrm{H}$ targets at several $Q^{2}$ values, while the HAPPEX experiment will make one final precision measurement at forward angle with a ${ }^{1} \mathrm{H}$ target at $Q^{2}=0.6 \mathrm{GeV}^{2}$. On the theoretical side, ultimate insight must await unquenched lattice QCD calculations with light, chiral quarks.

\section{NEUTRON SKIN OF A HEAVY NUCLEUS}

In a heavy nucleus such as ${ }^{208} \mathrm{~Pb}$, the difference between neutron radius $R_{n}$ and proton radius $R_{p}$ is believed to be several percent. Analogous to the classic measurement of $R_{p}$ via elastic electron electromagnetic scattering, $R_{n}$ can be measured via elastic electroweak scattering[16]. Experimentally, there is some controversy as to how well $R_{n}$ is known[17]; the best guess is $\sim 5 \%$.

The parity violating asymmetry in elastic scattering off a heavy spinless nucleus is proportional to the ratio of the neutron to proton form factors since the weak neutral current coupling of protons is much smaller than that of neutrons. A precise measurement of $R_{n}$ can have impact on nuclear theory, atomic parity violation and neutron star structure. Relativistic mean field models tend to favor larger neutron skins than nonrelativistic models because of a larger symmetry energy. The asymmetry measurement at an optimal value of $Q^{2}$ can pin down $R_{n}$ and help eliminate an entire class of models. Knowledge of $R_{n}$ at the $1 \%$ level can reduce the uncertainty in atomic structure of heavy isotopes that can cloud the interpretation of atomic parity violation measurements that test the Standard Model at the level of radiative corrections[18]. Finally, a precision $R_{n}$ measurement improves our understanding of the equation of state of neutron rich matter, which are important for constraining the structure of neutron stars[19].

With the improved control of helicity-correlated beam fluctuations in the running of the HAPPEX experiment, it has become feasible to make a measurement of $A_{\mathrm{PV}}$ in elastic scattering off a ${ }^{208} \mathrm{~Pb}$ target. For a beam energy of $850 \mathrm{MeV}$ and a scattering 
angle of $6^{\circ}$ and $Q^{2} \sim 0.01 \mathrm{GeV}^{2}$, the asymmetry of $0.5 \mathrm{ppm}$ would be measured with an uncertainty of $15 \mathrm{ppb}$. The experiment is scheduled to collect data in 2008 in Hall A at Jefferson Lab.

\section{LOW ENERGY WEAK MIXING ANGLE MEASUREMENTS}

The electroweak interaction has been tested to high precision at high energy colliders, especially by measurements of the properties of the $\mathrm{W}$ and $\mathrm{Z}$ bosons and their couplings to leptons and quarks. While all data are consistent with the standard model, experiments continue to probe for the indirect effects of new physics at the $\mathrm{TeV}$ scale by making more and more precise measurements of electroweak parameters[20].

Weak neutral current interactions at $Q^{2} \ll M_{Z}^{2}$ can probe for heavy $Z^{\prime}$ bosons or leptoquarks whose effects might be highly suppressed in measurements on the $Z$ pole. Since $\mathrm{Z}$ pole measurements are imaginary, there are no interference terms with new, real amplitudes. At low $Q^{2}$ on the other hand, interference effects might be measurable if sufficient accuracy is achieved[21]. The goal of low energy neutral current measurements is to reach a sensitivity on contact interactions at the $10 \mathrm{TeV}$ level, similar sensitivity to that of the highest energy colliders.

Three measurement techniques have reached such sensitivity. The weak charge measurement in atomic Cesium[23], the $\mathrm{NuTeV}$ neutrino deep-inelastic scattering measurement[22] and the measurement of $A_{\mathrm{PV}}$ in electron-electron (Møller) scattering[24] at SLAC. These measurements are used to set important new constraints on new parity-violating contact interactions at the $10 \mathrm{TeV}$ scale.

\section{Planned and Future Measurements at Jefferson Laboratory}

At sufficiently forward angles and low $Q^{2}$, the hadronic structure undertainty in the WNC elastic electron-proton amplitude becomes small enough such that one can measure the underlying coherent $2 u+d$ e-q amplitude combination to high precision. This combination is proportional to $1-4 \sin ^{2} \theta_{W}$, so that a $4 \%$ measurement of $A_{P V}$ would achieve a precision of $\delta\left(\sin ^{2} \theta_{W}\right)=0.0007$. Such a measurement has been proposed by the Qweak collaboration in Hall C at Jefferson Lab. Initial construction funds have been approved and the first data collection is projected for 2009.

With the upgrade of Jefferson Laboratory to $12 \mathrm{GeV}$, it is possible to achieve higher precision in the measurement of $A_{\mathrm{PV}}$ in Møller scattering. It might be possible to measure the $40 \mathrm{ppb}$ asymmetry to an accuracy of $2 \%$, which in turn would lead to a weak mixing angle measurement with an uncertainty of 0.00025 . At this level, the asymmetry competes directly with the best collider measurements and is sensitive to SUSY loops in the radiative corrections. In particular, the higher precision facilitates further stringent constraints on R-parity violating terms, with important implications for SUSY dark matter candidates[25]. 


\section{Fixed Target Møller Scattering at a Linear Collider}

One of the goals of precision electroweak physics measurements is to probe the scalar sector of the electroweak theory via ultra-precise measurements of fundamental electroweak parameters. For example, a measurement of the weak mixing angle $\sin ^{2} \theta_{\mathrm{W}}$ to a precision better than 0.00005 would measure the mass of the standard model Higgs boson to within $10 \%$ of itself[26]. Such a measurement is extremely challenging but several methods are being explored in future high energy collider experiments.

Measurements of such precision are very complementary if they are done at different energy scales. One promising method to reach such sensitivity at $Q^{2} \ll M_{Z}^{2}$ is with fixed target Møller scattering with the electron beam at a future linear collider[27]. The figure of merit of the measurement rises linearly with incident beam energy $E_{\text {beam }}$, a unique feature of Møller scattering due to the fact that the cross-section drops only linearly with $E_{\text {beam. }}$.

One idea is to develop a test beam and fixed target facility at a linear collider that would use the "exhaust" electron beam after the primary interaction at the collision point with the positron beam[28]. Thus, it would be possible to run fixed target experiments simultaneously with the electron-positron annihilation experiments. It is estimated the $\sin ^{2} \theta_{\mathrm{W}}$ would be measured with a precision of 0.00007 with physics runs of $10^{7} \mathrm{~s}$ at 250 $\mathrm{GeV} / \mathrm{c}^{2}$ and $500 \mathrm{GeV} / \mathrm{c}^{2}$.

\section{PARITY-VIOLATING DEEP INELASTIC SCATTERING AT JEFFERSON LABORATORY AT 11 GEV}

The upgrade of Jefferson Laboratory (Jlab) to $11 \mathrm{GeV}$ incident energy will allow precision measurements in parity-violating deep inelastic scattering (PV DIS). For the first time, high statistics can be accumulated at high $x \sim 0.7$, where $x$ is the fraction of the nucleon momentum carried by the struck quark. PV DIS provides access to novel aspects of nucleon structure, complementing and enhancing precision electromagnetic DIS studies.

$A_{P V}$ in DIS can be written as

$$
\begin{aligned}
A_{P V}= & Q^{2} \frac{G_{F}}{2 \sqrt{2} \pi \alpha}\left[a(x)+\frac{1-(1-y)^{2}}{1+(1-y)^{2}} b(x)\right], \\
& a(x) \equiv \Sigma_{i} f_{i}(x) C_{1 i} q_{i} / \Sigma_{i} f_{i}(x) q_{i}^{2} \\
& b(x) \equiv \Sigma_{i} f_{i}(x) C_{2 i} q_{i} / \Sigma_{i} f_{i}(x) q_{i}^{2} .
\end{aligned}
$$

Here, $C_{1 i}\left(C_{2 i}\right)$ are the weak vector(axial-vector) weak charges for the $i$ th quark flavor, $f_{i}(x)$ are parton distribution functions and $q_{i}$ are the electromagnetic charges. The $a(x)$ term arises from the product of the electron axial-vector coupling and the quark vector coupling and is typically the dominant term. For an isoscalar target such as deuterium, the dependence on structure largely cancels out in the $A_{P V}$ ratio of the weak and electromagnetic amplitudes:

$$
a(x)=\frac{6}{5}\left[\left(C_{1 u}-\frac{1}{2} C_{1 d}\right)+\text { corrections }\right]
$$




$$
b(x)=\frac{6}{5}\left[\left(C_{2 u}-\frac{1}{2} C_{2 d}\right) \frac{q(x)-\bar{q}(x)}{q(x)+\bar{q}(x)}+\text { corrections }\right],
$$

where $q(x)=u(x)+d(x)$. For scattering off the proton,

$$
a(x)=\left[\frac{u(x)+0.91 d(x)}{u(x)+0.25 d(x)}\right] .
$$

\section{Charge Symmetry Violation}

As can be seen from Eqn. 5, $a(x) \approx 1.15$ for an isoscalar target, independent of $x$. This results from the assumption of charge symmetry, where the $u$-quark distribution in the proton is the same as the $d$-quark distribution in the neutron, with a similar assumption for the proton $d$-quark distribution: $u^{p}=d^{n}$ and $d^{p}=u^{n}$. If $a(x)$ can be measured with high precision over a range of $x$ values, one is thus quite sensitive to charge symmetry violation (CSV). If one defines CSV parameters:

$$
\delta u(x)=u^{p}(x)-d^{n}(x) ; \quad \delta d(x)=d^{p}(x)-u^{n}(x),
$$

then the dependence on the parity-violating asymmetry for an isoscalar target is:

$$
\frac{\delta A_{P V}}{A_{P V}}=0.28 \frac{\delta u-\delta d}{u+d} \equiv 0.28 R_{C S V}
$$

While $R_{C S V}$ is known to be less than 0.01 for $x<0.4$ from neutrino DIS measurements[29], a bag model calculation suggests that $R_{C S V} \sim 0.01$ for $x \sim 0.4$ and rising to 0.02 for $x \sim 0.6$. At high $x$, knowledge of $u+d$ is limited. As $x \rightarrow 1$, if $u+d$ falls off more rapidly than $\delta u-\delta d$, then $R_{C S V}$ might rise to 0.1 at $x \sim 0.7$, which would be observable with a $1 \% A_{P V}$ measurement. Further, $R_{C S V}$ is quite unconstrained at large $x$. There is the tantalizing possiblity that $R_{C S V}$ in the moderate and high $x$ region is a factor of 3 bigger than above-mentioned values, which would be large enough to explain the $3 \sigma$ discrepancy in the neutrino-nucleon DIS measurement ( $\mathrm{NuTeV}$ anomaly)[29] and would be a very important discovery.

\section{$d / u$ at high $x$}

As can be seen from Eqn. 7 for PV DIS off the proton, $a(x)$ is quite sensitive to the ratio $d(x) / u(x)$. The value of $d / u$ as $x \rightarrow 1$ is a very important parameter to pin down in DIS physics. It is required in order to properly constrain fits of parton distribution functions and impacts predictions for QCD processes at high energy colliders.

More importantly, $d / u$ at high $x$ provides new information on important pieces of the nucleon wave function. There is empirical evidence that the minority quark in the nucleon is suppressed at high $x$, an intuitive notion in terms of a hyperfine interaction. While the $\mathrm{SU}(6)$ wave function would predict $d / u \sim 0.5$, simple $\mathrm{SU}(6)$-breaking arguments would predict $d / u \sim 0$. However, a perturbative QCD calculation predicts $d / u=0.2$ as $x \rightarrow 1[30]$. 
Currently, the best estimates of $d / u$ comes from ${ }^{2} \mathrm{H}$ DIS structure function data, but uncertainties in the ${ }^{2} \mathrm{H}$ wave-function limits the ability to discriminate between various predictions for $d / u[31]$. There are plans to measure $d / u$ via the ratio of ${ }^{3} \mathrm{H}$ and ${ }^{3} \mathrm{He}$ structure functions and also via measurements of deuteron structure functions with tagged slow recoiling protons.

A precise enough measurement of $a(x)$ for the proton at $x \sim 0.7$ would be able to distinguish between competing predictions for $d / u$ as $x \rightarrow 1$. The advantage of $A_{P V}$ measurements over other methods is that there are no nuclear corrections since the PV DIS measurement can be made on a proton target.

\section{Weak Mixing Angle Measurement}

The measurement of the PV DIS asymmetry to an accuracy of $0.5 \%$ at $Q^{2} \sim 5 \mathrm{GeV}^{2}$ and $x \sim 0.4$ for an isoscalar target such as deuterium, coupled with precise knowledge the parameter $a(x)$ from other experiments, the parameter $b(x)$ can be extracted with high accuracy for the first time. This measurement would be robust only if the comprehensive PV DIS program described above is carried out. Indeed, as can be seen from Eqn. 5, $a(x)$ is independent of $x$ and simply a function of $\sin ^{2} \theta_{W}$ under the assumption of charge symmetry and assuming that higher twist effects are either directly measured or constrained. The quantity $b(x)$ is also virtually free of structure function uncertainties at high $x$.

The measurement is interesting for several reasons. Firstly, it would test the WNC amplitude in the lepton-quark sector, where there is currently a $3 \sigma$ discrepancy in the $\mathrm{NuTeV}$ result. Secondly, combined with other measurements in elastic electron-proton scattering, precise constraints would be possible on the lesser known axial-vector quark couplings $C_{2 i}$. This would, among other things, provide complementary constraints on various models with new heavy $Z^{\prime}$ bosons[20].

\section{Experimental Equipment for PV DIS at high $x$}

To comprehensively address the physics topics discussed above experimentally, a series of $A_{P V}$ measurements in the range of 1 to $2 \%$ accuracy are required for the $x$ range from 0.3 to 0.7 , with a lever arm of a factor of 2 in $Q^{2}$ while keeping $W_{\min }^{2}>4$ and $Q_{\min }^{2}>1$. With the upgrade of Jlab, high luminosity with a beam energy of $11 \mathrm{GeV}$ becomes possible for the first time. However, to achieve sufficient statistics at the highest possible $Q^{2}$, a spectrometer with at least $50 \%$ acceptance in the azimuth is required.

A large magnet is required to shield the detectors from target photons and to sweep out low energy charged particles. Recent studies have indicated that a solenoid would be ideal for the required geometry, mainly the long target length and the large laboratory scattering angles. Feasibility studies and detailed simulations studies have just begun. It might be possible to use one of the existing collider detector solenoids after the corresponding experiments have been decommissioned. 


\section{SUMMARY}

Parity-violating electron scattering is a mature field and addresses fundamental questions in a variety of different topics. Recent results from the HAPPEX experiment at Jefferson Laboratory have indicated that the strange quark contribution to the nucleon charge and magnetization distributions is small. Future measurements will sharpen the limits and separate the electric and magnetic contributions over a range of $Q^{2}$ values. The E158 experiment at SLAC has produced the most precise measurement of the weak mixing angle at low energy. Future measurements at Jefferson Laboratory will lead to important new insights on the structure of the nucleon and yield new and more precise measurements of the weak mixing angle.

\section{ACKNOWLEDGMENTS}

It is a pleasure to thank the organizers for a stimulating meeting. The contributions of all the experimental collaborations discussed in this review are acknowledged. This work is funded in part by US Department of Energy Grant No. DE-FG02-88R40415-A018.

\section{REFERENCES}

1. Zel'dovich,Ya.B., J.Exptl.Theoret.Phys. (U.S.S.R.), 36, 1959, pp. 964-966.

2. Prescott,C.Y., et.al., Phys.Lett., B84, 1979, 524.

3. Kumar,K.S. and Souder,P.A., Prog.Part.Nucl.Phys., 45, 2000, pp. S333-S395.

4. Kaplan,D.B. and Manohar,A., Nucl.Phys., B310, 1988, 527.

5. McKeown,R.D., Phys.Lett., B219, 1989, 140.

6. Beck,D.H., Phys. Rev., D39, 1989, 3248.

7. Musolf,M.J., et.al., Phys. Rep., 239, 1994, 1, and references therein.

8. Beck,D.H. and Holstein,B.R., Int.J.Mod.Phys.,E10, 2001, pp. 1-41.

9. Spayde,D., et.al., Phys.Rev.Lett., 84, 2000, 1106; Hasty,R., et.al., Science, 290, 2000, 2117.

10. Aniol,K.A., et.al., Phys.Lett., B509, 2001, pp. 211-216.

11. Maas,F.E. et.al, Phys.Rev.Lett., 93, 2004, 022002; Maas,F.E. et.al, Phys.Rev.Lett., 94, 2005, 152001.

12. Armstrong,D.S., et.al, Phys.Rev.Lett. 95, 2005, 092001.

13. Aniol,K.A., et.al, Phys.Rev.Lett. 96, 2006, 022003; Aniol,K.A., et.al, Phys.Lett., B635, 2006, 275.

14. Lewis,R., et.al, Phys.Rev. D67, 2003, 013003.

15. Leinweber,D.B., et.al, Phys.Rev.Lett. 94, 2005, 212001; Leinweber,D.B., et.al, Phys.Rev.Lett. 97, 2006, 022001.

16. Donnelly,T.W., Dubach,J. and Sick,I., Nucl.Phys., A503, 1989, 589.

17. Horowitz,C.J., et.al., Phys.Rev., C63, 2001, 025501.

18. Pollock,S.J., Fortson,E.N. and Wilets,L., Phys.Rev., C46, 1992, 2587.

19. Horowitz,C.J. and Piekarweicz,J., Phys.Rev.Lett., 86, 2001, 5647.

20. Ramsey-Musolf,M.J., Phys.Rev., C60, 1999, 015501.

21. Kumar,K.S., et.al., Mod.Phys.Lett., A10, 1995, 2979.

22. Zeller,G.P., et.al., Phys.Rev.Lett., 88, 2002, 091802.

23. Wood,C.S., et.al., Science, 275, 1997, 1759.

24. Anthony,P.L., et.al, Phys.Rev.Lett., 95, 2005, 081601.

25. A. Kurylov, M.J. Ramsey-Musolf and S. Su, Phys. Rev. D68, (2003) 035008.

26. Fisher,P., Becker,U. and Kirkby,J., Phys.Lett., B356, 1995, pp. 404-408.

27. Kumar,K.S., "Fixed Target Møller Scattering at the NLC", in Snowmass 1996, New Directions for High-Energy Physics, 1996, pp. 1030-1034.

28. Keller,L., et.al., SLAC-PUB-8725 (2001). 
29. Londergan,J.T. and Thomas.A.W., hep-ph/0407247 (2004), and references therein.

30. Farrar,G.R. and Jackson,R.D., Phys.Rev.Lett. 43, 1979, 246.

31. Melnitchouk,W. et.al, Phys.Rev.Lett. 84, 2000, 5455; Melnitchouk,W. and Thomas,A.W., Phys.Lett. B377 1996, 11. 